\title{
Shards of SHARDS: emission line galaxies
}

\author{
Antonio Cava ${ }^{1}$, Víctor Villar ${ }^{1}$, Pablo G. Pérez-González ${ }^{1,2}$ \\ and the SHARDS Team ${ }^{3}$ \\ ${ }^{1}$ Departamento de Astrofísica, Facultad de CC. Físicas, Universidad Complutense de Madrid, \\ E-28040 Madrid, Spain, email: acava@fis.ucm.es \\ ${ }^{2}$ Associate Astronomer at Steward Observatory, The University of Arizona \\ ${ }^{3}$ SHARDS Team webpage: http://guaix.fis.ucm.es/ pgperez/SHARDS/team.html
}

\begin{abstract}
SHARDS (Survey for High-z Absorption Red \& Dead Sources; see Pérez-González et al. 2012) is an optical ultra-deep spectro-photometric survey with OSIRIS-GTC aimed at selecting and studying massive passively evolving galaxies at $z=1.0-2.3$ in GOODS-North. Nonetheless, the data quality allow a plethora of studies on galaxy populations, including Emission Line Galaxies (ELGs) about which we have started our first science verification project. The selection procedure, measurement and first analysis of ELGs in SHARDS, demonstrate the huge capability of the survey in providing physical information for a large sample of emitters, spanning from local $\mathrm{H} \alpha$ to high- $z$ Lyman- $\alpha$ emitters.
\end{abstract}

Keywords. galaxies: high-redshift — galaxies: fundamental parameters — galaxies: evolution

Spectroscopic observations are hard to obtain for large number of objects and very time consuming, however a powerful alternative can be found in (ultra-)deep medium/narrow-band imaging observations, which have been demonstrated to be useful to select emission-line galaxies with the faintest magnitudes. This Figure shows an example of the selection of ELGs with SHARDS data (Cava et al. 2012).

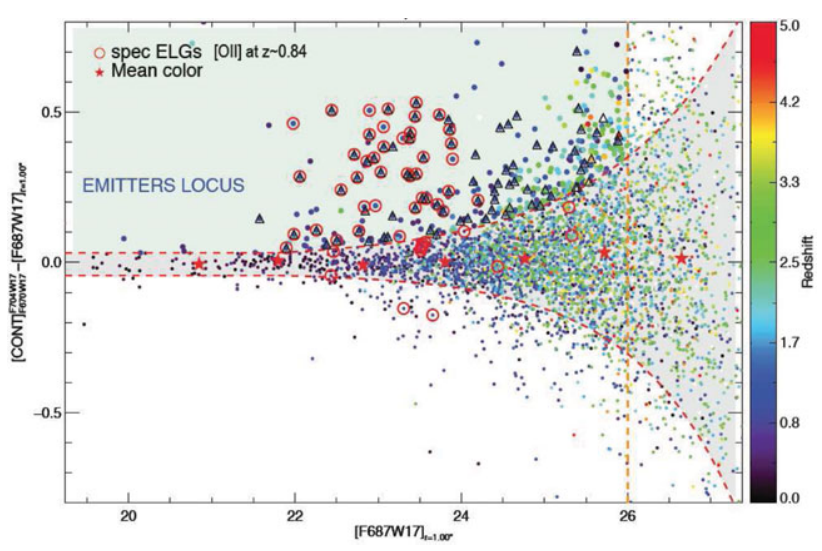

Sources with emission-lines lying inside the selected filter present an excess of flux compared to the average around it. In this particular example, we would expect to identify a galaxy population at $z \sim 0.84$ featuring an excess in the flux seen by the F687W17 filter due [OII] emission. Note that other lines could provide samples at different redshifts (e.g., Ly $\alpha$ at $z \sim 4.6$ or $[\mathrm{OIII}]$ at $z \sim 1.4$ ). After selecting the emitting galaxies (Emitters Locus) and identifying the corresponding detected lines using spec- and photo-z (respectively circles and triangles in Figure), we are able to compute line fluxes, equivalent widths and line luminosities. Then we build the observed luminosity function and study the physical properties of selected star forming galaxies (Cava et al., in prep.).

\section{References}

Cava, A. \& Pérez González, P. G., the SHARDS Team 2012, arXiv:1201.2177

Pérez-González, P. G., Cava, A., Barro, G., et al. 2012, ApJ accepted, arXiv:1207.6639 